\title{
Programmed cell death, proliferating cell nuclear antigen and p53 expression in mouse colon mucosa during diet-induced tumorigenesis
}

\author{
Mauro Risio ${ }^{\mathrm{a}, *}$, Ivana Sarotto ${ }^{\mathrm{a}}$, \\ Francesco Paolo Rossini ${ }^{\mathrm{b}}$, Harold Newmark ${ }^{\mathrm{c}}$, \\ Kan Yang ${ }^{\mathrm{c}}$ and Martin Lipkin ${ }^{\mathrm{c}}$ \\ ${ }^{a}$ Department of Pathology, Institute for Cancer \\ Research and Treatment, 10060 Candiolo-Torino, \\ Italy \\ ${ }^{\mathrm{b}}$ Department of Gastroenterology, Ospedale San \\ Giovanni Vecchio, 10123 Torino, Italy \\ ${ }^{\mathrm{c}}$ Strang Cancer Research Laboratory at the \\ Rockefeller University, New York, NY 10021, USA
}

Received May 2000

Accepted 16 November 2000

Western-style diets (WDs) trigger and sustain the early phases of tumorigenesis in mouse colon, and when continued throughout the life span lead to the development of dysplastic crypts. In order to evaluate the roles both of cell proliferation and programmed cell death (PCD) in WD-induced tumorigenesis, immunohistochemical detection of proliferating nuclear antigen (PCNA), in situ end labeling (TUNEL) of DNA breaks, and p53 protein were carried out in mouse colonic mucosa during prolonged feeding of two WDs. PCNA Labeling Index of colonic crypts was significantly higher in WD-treated animals than in controls only at the beginning of the nutritional study, the gap rapidly bridged by increased cell proliferation spontaneously occurring in the colonic mucosa during aging. A transient early homeostatic activation of PCD at the base of the crypt also was observed in WD groups. No changes in PCD were seen in the upper third of the crypt or in surface epithelium throughout the study, indicating that PCD in that colonic crypt segment produces a

\footnotetext{
${ }^{*}$ Corresponding author: Dr. Mauro Risio, Department of Pathology, Institute for Cancer Research and Treatment (IRCC), Strada Provinciale 142, km 3.95, 10060 Candiolo-Torino, Italy. Tel.: +39119933465; Fax: +39119933350; E-mail: mrisio@ mauriziano.it.
}

constant flux of cell loss, uninfluenced by homeostatic fluctuations. A major finding was an irreversible, progressive, agerelated decline of PCD at the crypt base in both control and treated animals that occurred during the second half of the rodents' life span. p53 protein was not immunohistochemically detected, suggesting that neither overexpression of wild-type nor mutated forms of the protein are involved in the above mentioned changes.

Keywords: Colon, carcinogenesis, cell proliferation, diet, programmed cell death

\section{Introduction}

We previously reported that Western-style diets (WDs) initiate and promote the early phases of tumorigenesis in mouse colonic mucosa [29]. The onset of dysplastic lesions induced by WDs is accompanied in grossly normal mucosa by complex and temporally linked changes in morphological features known to be related to tumor development: mitoses, indicative of cell proliferation; atypical mitoses and nuclei, indicative of genomic instability and clastogenic effect, respectively; apoptotic cells and apoptotic bodies as the end result of the process of programmed cell death (PCD) [29].

In order to highlight initial events in specific biological processes occurring in the colonic mucosa during the course of such nutritionally induced tumorigenesis, cell proliferation, PCD and their relationships were investigated using molecular markers for these cellular functions. Approaching cell differentiation in a similar way in the same model became quite informative [39].

Proliferating Cell Nuclear Antigen (PCNA) is an auxiliary DNA polymerase delta protein not expressed in $G_{0}$ and early $G_{1}$ cells, progressively expressed through the advanced $G_{1}$ phase and $S$ phase, followed 
by intermediate values in $\mathrm{G}_{2}$ and $\mathrm{M}$ [19]. PCNA is an effective molecular marker of cell proliferation, above all when used in immunohistochemistry of methanolfixed tissues [11]. MIB1 antibody is even more effective than PCNA in detecting cycling cells [28], but it does not react with $\mathrm{Ki}-67$ antigen in mouse nuclei [35].

One of the early events of PCD is the cleavage of nuclear DNA into oligonucleosome-sized fragments [38] which can be nick-end labeled in histological sections with biotinylated nucleotide, introduced by exogenous terminal deoxynucleotidyl transferase (TdT) and then stained using avidin-conjugated peroxidase (TUNEL technique) [13]. In situ labeling of DNA strand breaks detects a PCD-related phenomenon which is earlier and is thought to have a longer duration than apoptotic morphological changes: it is therefore supposed to amplify the possibility of identifying cells engaged in PCD [14].

PCD is an effective process aimed at regulating tissue homeostasis and deleting cells that have sustained irreversible genotoxic damage [18]. Functional p53 protein is required by cells with genotypic alterations to trigger their cell death program [5] or, alternatively, to inhibit progression through $G_{1}$ into $S$ phase [8]. In the mouse intestine, however, PCD is the prevailing p53-mediated response to genotoxic damage [6].

With this theoretical background, relationships of these biological processes were studied with PCNA and p53 immunohistochemistry, and with TUNEL technique performed in the WD-induced tumorigenesis model previously analyzed for histology.

\section{Material and methods}

\subsection{Animals and diets}

180 C75/BL6J mice (90 males and 90 females) at 3 weeks of age were fed AIN-76A semisynthetic control diet for 3 weeks for acclimatization and then randomly divided to ad libitum feeding of control diet (CD) (AIN-76A) or experimental diets simulating two WDs (high fat and phosphate, low calcium and vitamin D). American Blend Fat was used in WD A, corn oil in WD B. The components of the three diets are detailed in [29]. At 12, 19, 27, 52, 72 and 104 weeks after the start of the experimental feeding 10 animals (5 males and 5 females) in each experimental group were sacrificed. To avoid the interference of circadian variations in colonic cell proliferation, all animals were sacrificed between 8 and 9 AM.

\subsection{Tissue specimens}

The colons were removed and, in order to avoid artifacts in TUNEL staining [15], immediately fixed in $80 \%$ ethanol for $24 \mathrm{~h}$. Cross sections of the descending distal colon with grossly normal appearing mucosa were taken and transferred to $95 \%$ ethanol for processing. The tissues were dehydrated in absolute ethanol and embedded in paraffin. Four- $\mu \mathrm{m}$ sections were dewaxed and rehydrated. Endogenous peroxidase activity was blocked by incubating the sections in $3 \% \mathrm{H}_{2} \mathrm{O}_{2}$. At the end of the procedures, the slides were weakly counterstained with hematoxylin, dehydrated, clarified, mounted in Entellan (Merck, Darmstadt, Germany), and examined under a standard light microscope. Immunostaining for PCNA was done with the PC 10 anti-PCNA Mab (Dako A/S, Glostrup, Denmark) diluted $1: 50$, followed by incubation with biotinylated antimouse immunoglobulins. The CM1 anti-p53 polyclonal rabbit antiserum (Biogenex, San Ramon, CA), reacting with wild-type and most mutant forms of p53 protein, was used for $60 \mathrm{~min}$ at RT after microwaving the sections at $700 \mathrm{~W}(2 \times 5 \mathrm{~min})$ in Antigen Retrieval Citra Solution (BioGenex, San Ramon, CA); incubation with biotinylated anti-rabbit immunoglobulins followed. The conventional peroxidase conjugated streptavidin was employed to show the reactions.

\subsection{TUNEL}

After digestion of nuclear proteins by proteinase $\mathrm{K}$ (Sigma BioSciences, St. Louis, MO), $20 \mu \mathrm{g} / \mathrm{ml}$ at RT for $5 \mathrm{~min}$, the slides were immersed in TdT buffer (30 mM TRIZMA base, $\mathrm{pH}$ 7.2, $140 \mathrm{mM}$ sodium cacodylate, $1 \mathrm{mM}$ cobalt chloride). TdT 0.15 e.u. $/ \mathrm{ml}$ (Sigma Biosciences, St. Louis, MO) and Bio-11-dUTP (Enzo Diagnostic, Farmingdale, NY) in TdT buffer were then added to cover the sections, incubated for $60 \mathrm{~min}$ in a moist chamber at $37^{\circ} \mathrm{C}$. The reaction was terminated by transferring the slides to TB buffer for $15 \mathrm{~min}$ at RT. The sections were then rinsed twice with DDW for $3 \mathrm{~min}$, covered with $2 \%$ acqueous solution of BSA for 10 min at RT, rinsed in DDW, immersed in $0.01 \mathrm{M}$ PBS pH 7.2 with Tween 20 for $5 \mathrm{~min}$, covered and incubated with peroxidase conjugated streptavidin for $15 \mathrm{~min}$ at $37^{\circ} \mathrm{C}$, rinsed in PBS with $0.1 \%$ Tween 20 and finally stained with diaminobenzidine solution for about $30 \mathrm{sec}$. 


\subsection{Scoring methods}

Four hundred longitudinally oriented crypt columns were examined in each animal. All labeled nuclei were regarded as positive, irrespective of their staining intensity. Their number and distribution were evaluated by dividing each crypt column in three longitudinal compartments from the basal third of the colonic crypt (compartment 1) to the middle third (compartment 2) and the upper third reaching the mouth of the crypt, including the surface epithelium (compartment 3 ). The PCNA Labeling Index (LI), the p53 Index, and the TUNEL Index (i.e., the per cent ratio between labeled and total nuclei) were used to provide a quantitative assessment of the results in the whole crypt and in each compartment.

\subsection{Statistics}

Result were expressed as means \pm SEM. Student's test for unpaired data was primarily used to determine the significance of differences between the means of indices for the three group of animals. Wilcoxon's rank sum test was used when the data were abnormally distributed, as seen in the compartment 3. Statistical significance was assumed for $P<0.05$.

\section{Results}

PCNA LI progressively increased in the whole crypt column, in compartment 1 and 2 (Figs 1A, 2A, 2B, respectively) during the first half of the rodents' life span in CD fed mice, remarkably in the period 12-27 weeks. Such increase was not seen in WD groups where relatively high and stable PCNA LI values were already present at the beginning of the nutritional study: as a consequence significantly higher PCNA LIs characterized WD A and WD B groups compared to controls at 12 weeks. Since the TUNEL Index per column was mostly unmodified in all groups throughout the rodents' life span (Fig. 1B), the only gap in PCNA LI/TUNEL Index ratio between $\mathrm{CD}$ and WD groups was noted at 12 weeks (Fig. 1C).

A significant but transient increase of TUNEL Index at the base of the crypt (compartment 1) was associated with WD feeding at 12 weeks (Fig. 3A).

The most striking event occurring at the base of the crypt was the progressive decrease of TUNEL Index in both control and treated animals, beginning from the 27 th week (Fig. 3A). The percentages of de-
A

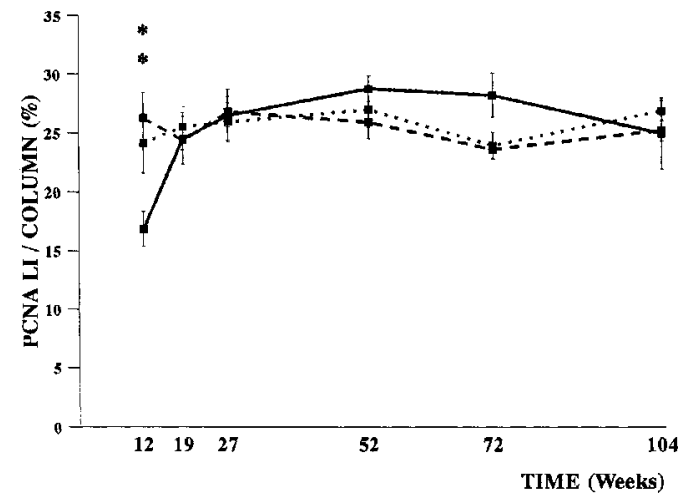

B

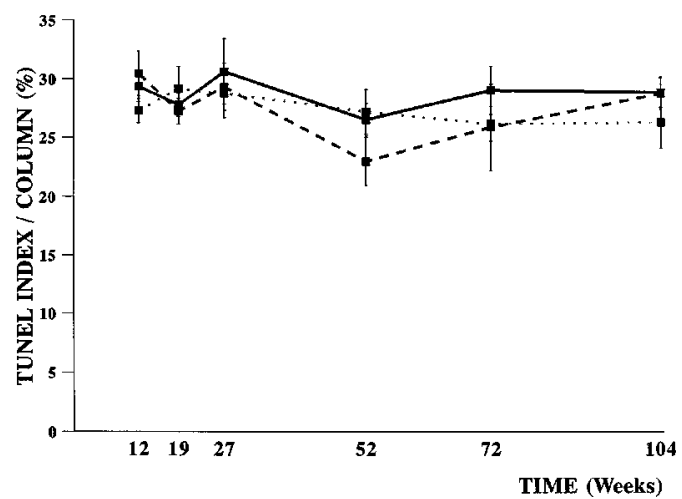

c

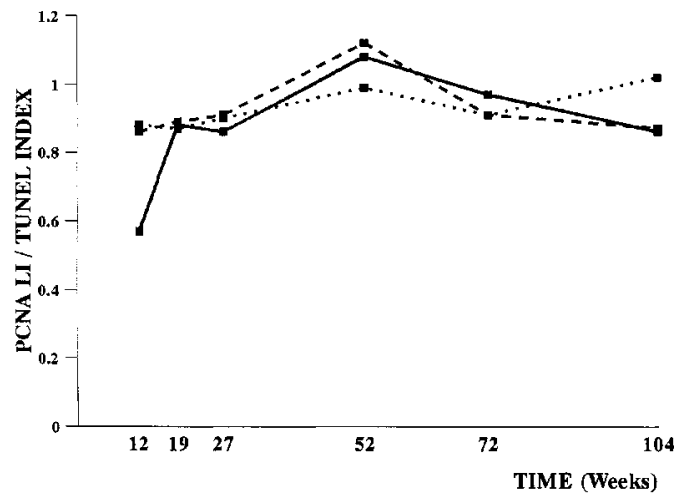

Fig. 1. PCNA LI (A), TUNEL Index (B) in the whole crypt column, and PCNA LI / TUNEL Index ratio (C) during the rodents' life span. (Control Diet, CD: _ ; Western Diet A, WD A: ...; Western Diet B, WD B: - - - ; vertical bars: SEM; $* P<0.05$ vs controls.)

crease in compartment 1 TUNEL Index during the period 27-104 weeks were $80.65 \%, 87.54 \%, 78.48 \%$ for $\mathrm{CD}$, WD A, WD B group, respectively, the major negative slope ( $-60 \%$ on the average) being in the middle of the rodents' life span (27-52 weeks). These findings accompanied the development and progressive increase of atypical nuclei and whole crypt 
A

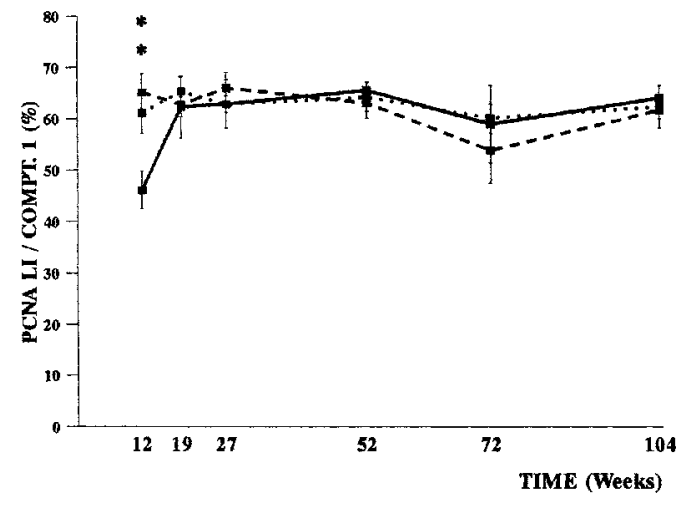

B

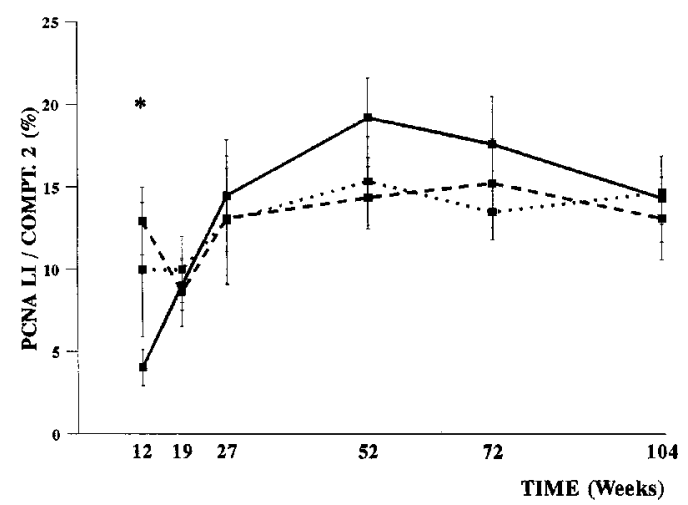

C

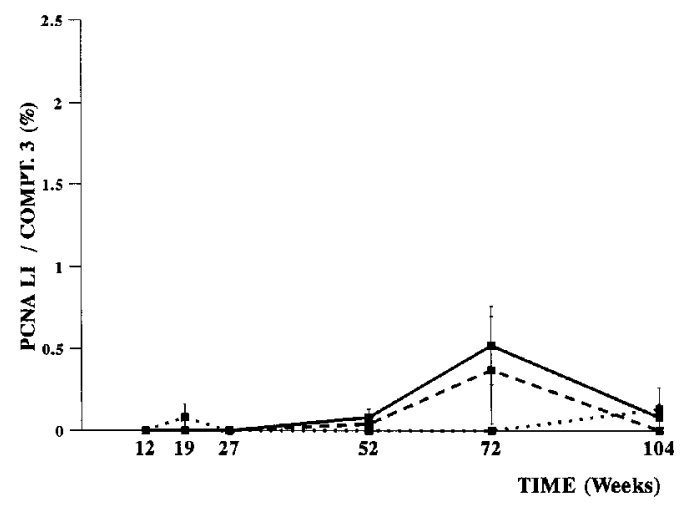

Fig. 2. PCNA LI in compartment 1 (A), compartment 2 (B), and compartment 3 (C). (Control Diet, CD: —; Western Diet A, WD A: ...; Western Diet B, WD B: --.-; vertical bars: SEM; $* P<0.05$.)

dysplasia in the colon, which occurred in the same study [29].

No intergroup and intragroup significant variations were seen in both PCNA and TUNEL Indices in compartment 3 (Figs 2C and 3C).

p53 immunoreactivity was never detected, either in WD groups or in controls.
A

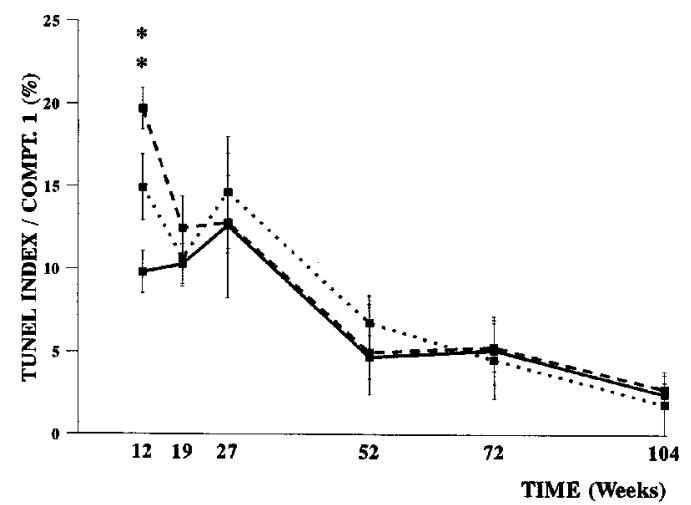

B

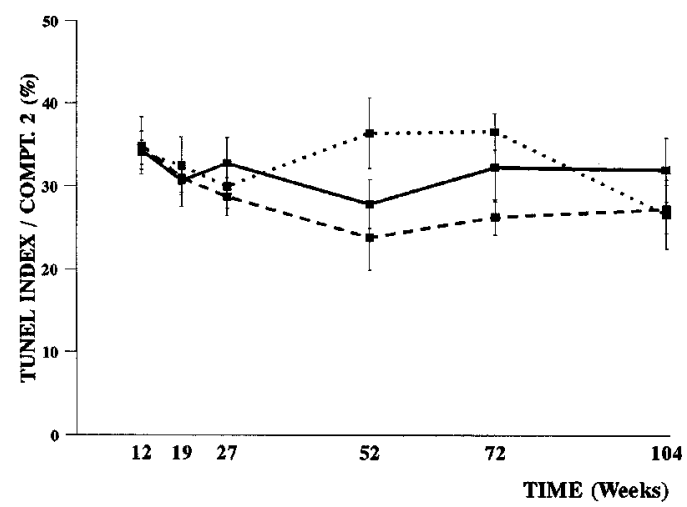

C

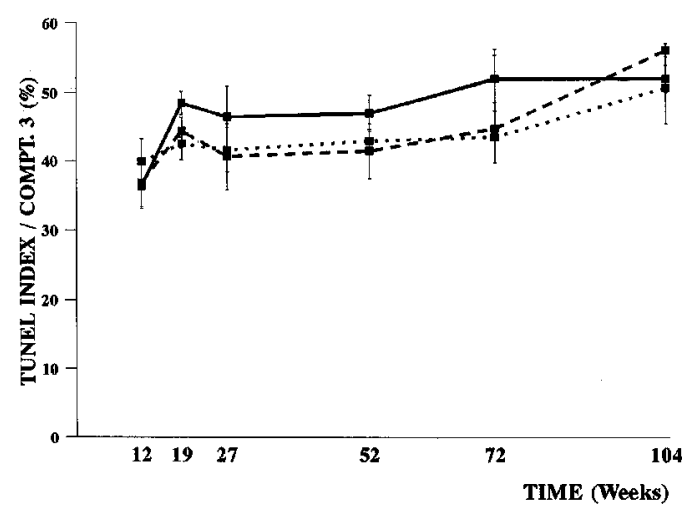

Fig. 3. TUNEL Index in compartment 1 (A), compartment 2 (B), and compartment 3 (C). (Control Diet, CD: __; Western Diet A, WD A: ...; Western Diet B, WD B: -.--; vertical bars: SEM; $* P<0.05$.)

\section{Discussion}

Cell proliferation changes in the colonic mucosa are considered a hallmark of early premorphological stages of colonic tumorigenesis [26, p. 155]. Hyperproliferation induced by WDs in short term studies [24] corresponds to the high PCNA LI observed in this 
study, in WD-treated groups during the first 12 weeks of feeding. Interestingly, the differences in PCNA LIs between WD groups and controls were rapidly cancelled by the spontaneous and progressive increase of cell proliferation in control animals, remarkably in compartment 2 , during the period $12-52$ weeks. Increased cell proliferation in the colonic mucosa in relation to age has also been previously observed in both rodents [17] and humans [30], and has to some extent been correlated with an age-related risk of cancer. In keeping with results in the present study, the effect of WDs on colonic mucosa was not the induction of a peak of hyperproliferation, but rather the anticipation and/or acceleration of an age-related up-regulation of cell proliferation.

In addition to DNA replication, PCNA has a role in several functions, among which DNA repair is well known [27,37]. As a consequence PCNA expression in this nutritional experiment could be linked with both DNA replication in proliferating cells and DNA repair of the WD-induced genotoxic damage. Hirose et al. [16] have shown that PCNA immunostaining of colonic epithelium after azoxymethane exposure depends both on DNA repair and cell proliferation. That does not seem to be the case in this investigation, however, since PCNA LIs are similar except at 12 weeks, in all groups, even at periods (19 weeks, 104 weeks) in which the mucosa harbours atypical nuclei in great number, these representing the morphological evidence of WD-induced DNA damage [29].

A significant imbalance between cell proliferation and cell death in the colonic mucosa has not been found in this model. The PCNA LI/TUNEL Index ratio were closely linked over a narrow range throughout the rodents' life span in both WD groups, and values of this ratio paralleled controls, except at the beginning of the experiment. Since at that point PCD levels were roughly the same in all three groups, the low value of PCNA LI/TUNEL Index ratio in controls at the 12th week is ascribable to the absence of the WD-induced activation of proliferation, in accordance with the view that the first event induced by WDs is a derangement in cell-cycle regulation rather than PCD misregulation [29].

An increase in cell proliferation without an effective homeostatic response should lead to the expansion of tissue size. Effectively colon mucosal hyperplasia (i.e., the elongation of the crypt by increased number of epithelial cells in crypt columns) has been shown to occur, after WD treatment, in short-term studies [23] and, at the 12 th week in the same mucosa investigated in the current study [29]. The mechanisms responsible for the spontaneous recovery of the steady state in the homeostatic trophism of the mucosa, which have not been previously elucitated, should be sought among the subtle alterations affecting PCD topographic distribution.

Although in this study PCD was almost constant in the whole colonic crypt [29], remarkable changes were seen when evaluating TUNEL Index in the different sectors of the crypt. A significant increase in the percentage of TUNEL positive cells at the base of the crypt was found in WD-treated animals, limited at the 12th week. Even if quantitatively inadequate to modify the PCNA LI/TUNEL Index ratio at that point, such a transient and localized activation of PCD is likely to be induced by DNA-damaged cells, since a clastogenic effect is exerted early by WDs in this nutritionally induced tumorigenesis [29]. The deletion of genetically aberrant cells also includes cells derived from abnormal symmetrical stem cell division at the base of the crypt. Normally, stem cell division is asymmetric [21] and the intervention of PCD on this perturbed system could act to prevent hyperplasia or to restore normal tissue size [33]

The activation of PCD at the crypt base was unaccompanied by any p53 immunoreactivity in the mucosa. There is evidence that spontaneous apoptosis in the crypt is p53-independent, whereas the apoptotic response following irradiation is p53-dependent [6]: in that case a significant increase in p53-positive nuclei occurs throughout the colonic crypt a few hours after irradiation [22], owing to the posttranslational stabilization of the p53 protein. The lack of p53 immunostaining in our model is consistent with the hypothesis that p53 protein is not involved in recognition of early DNA damage induced by WDs in colonic mucosa as well as in the subsequent PCD activation. It should be taken into account, however, that wild-type p53, differently from the mutated counterpart, has a short halflife and is immunohistochemically detectable only if significantly overexpressed [2]: the clastogenic effect exerted by WD could be too scanty to elicit a p53 response above the immunodetection threshold. Nutritional experiments in p53 mutated mouse are required for the final explanation of this result.

No further differences in PCD distribution among the groups were seen, and in addition TUNEL Index in the various compartments of the crypt was coincident for treated and control animals at several points. In particular, at the upper third of the crypt and in the surface epithelium PCD levels were not modified throughout the entire duration of the experiment in all groups. 
The different behaviours of PCD at the base and at the top of the crypt indicates different biological roles exerted by PCD in these sectors. In human colonic mucosa two different morphological patterns of apoptosis exist, "engulfment" and "extrusion" [33], localized at the base and at the luminal surface mucosa, respectively. Apoptotic activation in the basal regions is elicited by exposure to genotoxic or cytotoxic agents, whereas in the surface epithelium apoptosis is damageindependent, linked to cell senescence [25] and likely to be triggered and substained by modifications occurring in enterocyte-cell matrix interactions $[10,34]$. There is also mathematical evidence that apoptosis inside the crypt rather than shedding into the lumen from the surface epithelium, is the actual regulator of homeostasis in the gut [15]. According to Strater et al. [33], two distinct routes of apoptosis are available inside the colonic crypt; alternatively, a single apoptotic program could be hypothesized, which is under different temporal, microenvironmental, and genetic controls in the various sectors of the crypt $[25,33]$. Taken together all these data support the unifying model suggested by Budz [4, p. 165] to explain the homeostatic control of the epidermal renewal: homeostasis is the result of the plastic and modulable balancing of the interactions between mitoses and apoptosis ("Regulated cell death") in the stratum intermedium, whereas PCD in superficial, terminally differentiated and keratinized cells produces a constant cell loss uninfluenced by homeostatic fluctuations.

The striking and progressive decrease of PCD at the base of the crypt during the period 27-104 weeks was to some extent already observed in a previous study, as a fall of apoptotic cells in the middle of the rodent's life span [29]. That phenomenon, occurring simultaneously with the onset of gross dysplastic lesions in the colon [29], was thought to allow the clonal expansion of cells bearing WD-induced DNA damage. The depletion of apoptotic/PCD activity occurred in the same manner in both control and treated animals and seemed therefore unrelated to the nutritional effects and linked to physiological events. The results of the current study further pinpoint a progressive and irreversible PCD decline, indicative of an age-related process. In effect, the aging process has been experimentally demonstrated to be the consequence of progressive failure of cells within an organism to retain sensitivity to apoptotic signals, and in vivo data suggest that PCD is less efficient with increasing age [36, p. 377]. Relationships between PCD and aging in human colon are not yet defined, although decreasing apoptosis with age in a
Western population exposed to bile acids has been suggested [12].

Two major points differentiate the observed PCD and apoptosis attenuation. Fall of apoptotic cells was seen in compartment 2, i.e., in the middle third of the crypt, whereas the decrease of TUNEL positive cells was localized at the base. The duration of the apoptotic process, however, is unknown and cells engaged in apoptosis are simultaneously migrating toward the mucosal surface [25]: it is therefore possible that cells showing DNA breaks in compartment 1 could be morphologically identified as apoptotic only after their migration into compartment 2 . The most remarkable divergence concerns the evolution of the two phenomena. In spite of the irreversible impairment of PCD, as shown here by the progressive decrease of TUNEL Index, the apoptotic function was rapidly and completely restored after a transient fall at 52 weeks [29]. Although the correlation between TUNEL positive and apoptotic cells turned out to be quite strong in human colon [32] it is well known that DNA fragmentation is not uniquivalently linked with apoptosis $[1,31]$. Not all the cells with DNA breaks proceed to morphological evidence of apoptosis, and 17-35\% of apoptotic cells of intestinal crypt are TUNEL negative [25]. Basically, DNA fragmentation can be induced by radiation exposure, necrotic degradation, DNA isolation besides apoptosis $[1,9,25]$; on the other hand apoptosis can be activated and substained in the absence of internucleosomal DNA cleavage [7]. The results of the current study, showing a preserved apoptotic function in the presence of impaired DNA fragmentation, suggest that a physiological shift occurs, in the middle of rodents' life span, from nuclear endonuclease, to a different and unknown mechanism of activation of apoptosis.

In conclusion, our results indicate that Westernstyle diets induce early up-regulation of mucosal cell proliferation, accompanied by a homeostatic, p53independent increase of PCD at the base of the crypt. Of major importance, an age-related progressive decrease of PCD in the same crypt segment is a salient feature during the later half of the rodents' life span, as tumorigenic WD-induced dysplasia appears and accumulates in the colonic mucosa. The PCD above, however, cannot be used as an intermediate biomarker of colonic tumorigenesis since it is present to the same extent in both controls and WD groups. Nevertheless, changes in PCD topographic distribution have been described in the mucosa of patients with familial adenomatous polyposis [3]. Further investigations are required to verify if these changes are specifically linked with a familial rather than a dietary pathway of colorectal tumorigenesis [20]. 


\section{Acknowledgements}

This work was supported in part by the Italian Association for Cancer Research (AIRC), US National Cancer Institute Award NOI-CN-15363, and the Ann Eden Woodward Foundation.

\section{References}

[1] B. Ansari, P.J. Coates, B.D. Greenstein and P.A. Hall, In situ end-labelling detects DNA strand breaks in apoptosis and other physiological and pathological states, J. Pathol. 170 (1993), 1-

[2] H. Battifora, p53 immunohistochemistry: a word of caution, Hum. Pathol. 25 (1994), 435-437.

[3] A. Bedi, P.J. Pasricha, A.J. Akhtar, J.P. Barber, G.C. Bedi, F.M. Giardiello, B.A. Zehnbauer, S.R. Hamilton and R.J. Jones, Inhibition of apoptosis during development of colorectal cancer, Cancer Res. 55 (1995), 1811-1816.

[4] P.E. Budz, Epidermal homeostasis: a new model that includes apoptosis, in: Apoptosis II: The Molecular Basis of Apoptosis in Disease, L.D. Tomei and F.O. Cope, eds, Cold Spring Harbor Laboratory Press, Plainview, New York, 1994.

[5] A.R. Clarke, C.A. Purdie, D.J. Harriason, R.G. Morris, C.C. Bird, M.L. Hooper and A.H. Wyllie, Thymocyte apoptosis induced by p53-dependent and independent pathways, Nature 362 (1993), 849-852.

[6] A.R. Clarke, S. Gledhill, M.L. Hooper, C.C. Bird and A.H. Wyllie, p53 dependence of early apoptotic and proliferative response within the mouse intestinal epithelium following g-irradiation, Oncogene 9 (1994), 1767-1771.

[7] G.M. Cohen, X.-M. Sun, R.T. Snowden, D. Dinsdale and D.N. Skilleter, Key morphological features of apoptosis may occur in the absence of internucleosomal DNA fragmentation, Biochem. J. 286 (1992), 331-334.

[8] W.S. El-Deiry, T. Tokino, V.E. Velculescu, D.B. Levy, R. Parson, J.M. Trent, D. Lin, W.E. Mercer, K.W. Kinzler and B. Vogelstein, WAF1, a potential mediator of p53 tumor suppression, Cell 75 (1993), 817-825.

[9] H. Enright, R.P. Hebbel and K.A. Nath, Internucleosomal cleavage of DNA as the sole criterion for apoptosis may be artifactual, J. Lab. Clin. Med. 124 (1994), 63-68.

[10] S.M. Frisch and H. Francis, Disruption of epithelial cell-matrix interactions induces apoptosis, J. Cell. Biol. 124 (1994), 619626.

[11] P. Galand and C. Degraef, Cyclin/PCNA immunostaining as an alternative to tritiated thymidine pulse labeling for marking $S$ phase cells in paraffin sections from animals and human tissues, Cell. Tissue Kinet. 22 (1989), 283-292.

[12] H. Garewal, H. Bernstein, C. Bernstein, R. Saampliner and C. Payne, Reduced bile acid-induced apoptosis in "normal" colorectal mucosa: a potential biological marker for cancer risk, Cancer Res. 56 (1996), 1480-1483.

[13] Y. Gavrieli, Y. Sherman and S.A. Ben-Sasson, Identification of programmed cell death in situ via specific labeling of nuclear DNA fragmentation, J. Cell. Biol. 119 (1992), 493-501.
[14] W. Gorczyca, T. Tuziack, A. Kram, M.R. Melamed and Z. Darzynkievwicz, Detection of apoptosis-associated DNA strand breaks in fine needle aspiration biopsies by in situ end labeling of fragmented DNA, Cytometry 15 (1994), 169-175.

[15] P.A. Hall, P.J. Coates, B. Ansari and D. Hopwood, Regulation of cell number in the mammalian gastrointestinal tract: the importance of apoptosis, J. Cell. Sci. 107 (1994), 3569-3577.

[16] Y. Hirose, N. Yoshimi, A. Hara, T. Tanaka and H. Mori, Early alterations of apoptosis and cell proliferation in azoxymethaneinitiated rat colonic epithelium, Jap. J. Cancer Res. 87 (1996), 575-582.

[17] P.R. Holt and K.-Y. Yeh, Colonic proliferation is increased in senescent rats, Gastroenterology 95 (1988), 1556-1563.

[18] J.F.R. Kerr, C.M. Winterford and B.V. Harmon, Apoptosis. Its significance in cancer and cancer therapy, Cancer 73 (1994), 2013-2026.

[19] P. Kurki, M. Vanderlaan, F. Dolbeare, J. Gray and M.E. Tan, Expression of proliferating nuclear antigen (PCNA/Cyclin) during the cell cycle, Exp. Cancer Res. 166 (1986), 209-212.

[20] M. Lipkin, K. Yang, W. Edelman, H. Newmark, K.-H. Fan, M. Risio and R. Kucherlapati, Inherited and and acquired risk factors in colonic neoplasia and modulation by chemopreventive interventions, J. Cell. Biochem. 25S (1996), 136-141.

[21] M. Loeffer, A. Birke, D. Winton and C.S. Potten, Somatic mutations, monoclonality and stochastic models of stem cells organisation in the intestinal crypt, J. Theor. Biol. 160 (1993), 471-491.

[22] A.J. Merritt, C.S. Potten, C.J. Kemp, J.A. Hickman, A. Balmain, D.P. Lane and P.A. Hall, The role of p53 in spontaneous and radiation-induced apoptosis in the gastrointestinal tract of normal and p53-deficient mice, Cancer Res. 54 (1994), 614617.

[23] H.L. Newmark, M. Lipkin and N. Maheshwari, Colonic hyperplasia and hyperproliferation induced by a nutritional stress diet with four components of Western-style diet, J. Natl. Cancer Inst. 82 (1990), 491-496.

[24] H. Newmark, M. Lipkin and N. Maheshwari, Colonic hyperproliferation induced in rats and mice by nutritional stress diets containing four components of a human Western-style diet (series 2), Am. J. Clin. Nutr. 54 (1991), 2095-2145.

[25] C.S. Potten, What is an apoptotic index measuring? A commentary, Br. J. Cancer 74 (1996), 1743-1748.

[26] M. Risio, Mucosa cell proliferation in colorectal neoplasia, in: Recent Progress in Colorectal Cancer: Biology and Management of High Risk Groups, F.P. Rossini, H.T. Lynch and S.J. Winawer, eds, Elsevier Science Publishers B.V., Amsterdam, 1992.

[27] M. Risio, G.L. Candelaresi and F.P. Rossini, Bromodeoxyuridine uptake and proliferating cell nuclear antigen expression throughout the colorectal tumor sequence, Cancer Epidemiol. Biomarkers \& Prev. 2 (1993), 363-367.

[28] M. Risio, Methodological aspects of using immunohistochemical cell proliferation biomarkers in colorectal carcinoma chemopreventiom, J. Cell. Biochem. 19S (1994), 61-67.

[29] M. Risio, M. Lipkin, H. Newmark, K. Yang, F.P. Rossini, V.E. Steele, C.W. Boone and G.J. Kelloff, Apoptosis, cell replication, and western-style diet-induced tumorigenesis in mouse colon, Cancer Res. 56 (1996), 4910-4916. 
[30] L. Roncucci, M. Ponz de Leon, A. Scalmati, G. Malagoli, S. Pratissoli, M. Perini and N. Chahin, The influence of age on colonic epithelial cell proliferation, Cancer 62 (1988), 2373 2377.

[31] K. Schulze-Osthoff, H. Wakzcak, W. Droge and P.H. Krammer, Cell nucleus and DNA fragmentation are not required for apoptosis, J. Cell. Biol. 127 (1994), 15-20.

[32] F.A. Sincrope, G. Roddey, T.J. McDonnell, Y. Shen, K.R. Cleary and L.C. Stephens, Increased apoptosis accompanies neoplastic development in the human colorectum, Clin. Cancer Res. 2 (1996), 1999-2006.

[33] J. Strater, A.R. Gunthert and P. Moller, In situ detection of enterocytic apoptosis in normal colonic mucosa and in familial adenomatous polyposis, Gut 37 (1995), 819-825.

[34] J. Strater, U. Wedding, T.F. Barth, K. Koretz, C. Elsing and P. Moller, Rapid onset of apoptosis in vitro follows disruption of beta 1-integrin/matrix interactions in human colonic crypt cells, Gastroenterology 110 (1996), 1776-1784.
[35] K.P. Teter, D.C. Holloway and G.E. Sandusky, Assessment of PCNA (19A2) and Ki-67 (MIB1) cell proliferation markers in formalin fixed tissues, The J. Histotechnol. 18 (1995), 99-104.

[36] L.D. Tomei, F.O. Cope and P.J. Barr, Apoptosis: aging and phenotypic fidelity, in: Apoptosis II: The Molecular Basis of Apoptosis in Disease, L.D. Tomei and F.O. Cope, eds, Cold Spring Harbor Laboratory Press, Plainview, NY, 1994.

[37] L. Toschi and R. Bravo, Changes in cyclin/PCNA distribution during DNA repair synthesis, J. Cell. Biol. 107 (1988), 16231628 .

[38] P.R. Walker, V.M. Weaver, B. Lach, J. Leblanc and M. Sikorska, Endonuclease activities associated with high molecular weight and internucleosomal DNA fragmentation in apoptosis, Exp. Cell. Res. 213 (1994), 100-106.

[39] K. Yang, K. Fan, H. Newmark, D. Leung, M. Lipkin, V.E. Steele and G.J. Kelloff, Cytokeratin, lectin, and acid mucin modulation in differentiating colonic epithelial cells of mice after feeding western-style diets, Cancer Res. 56 (1996), 4644-4648. 


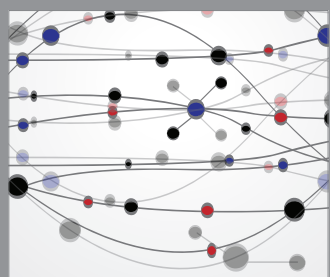

The Scientific World Journal
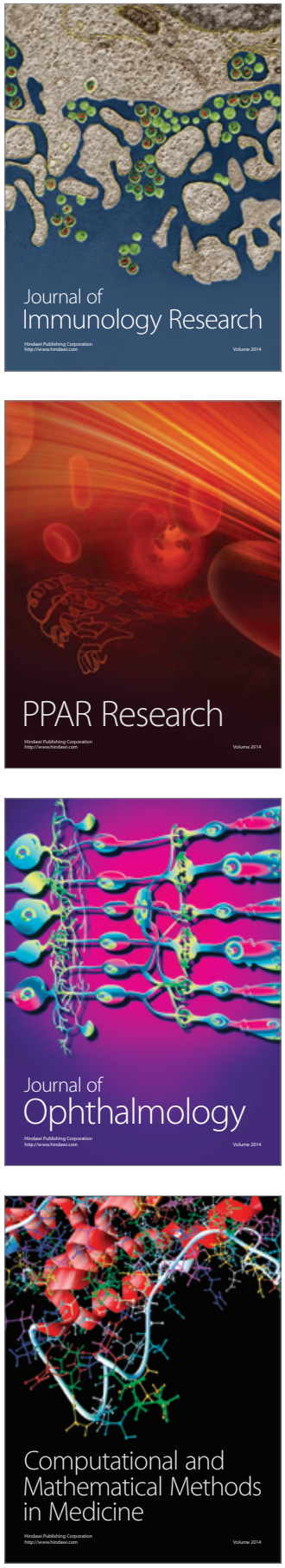

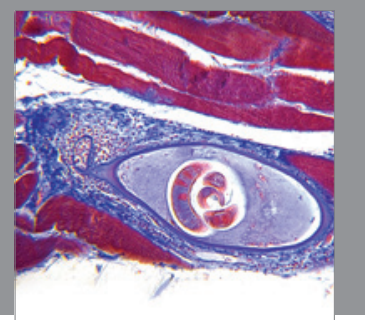

Gastroenterology

Research and Practice
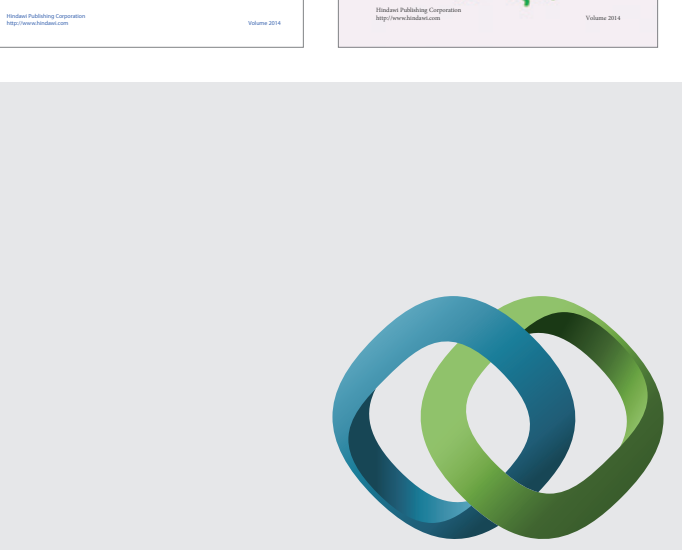

\section{Hindawi}

Submit your manuscripts at

http://www.hindawi.com
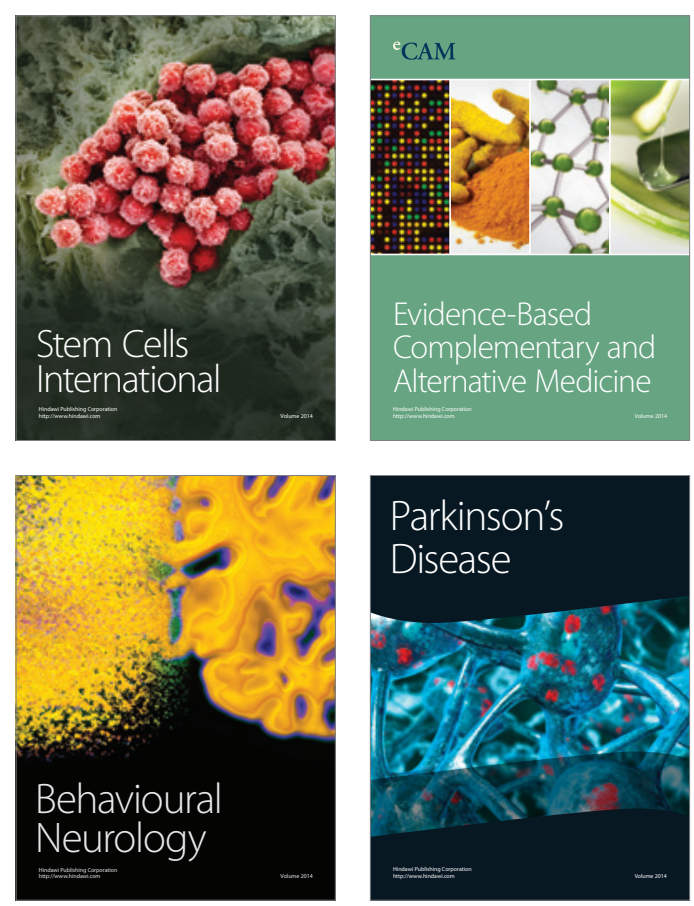

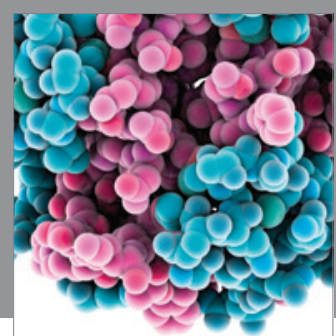

Journal of
Diabetes Research

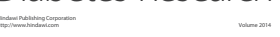

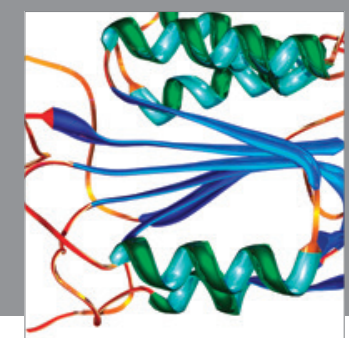

Disease Markers
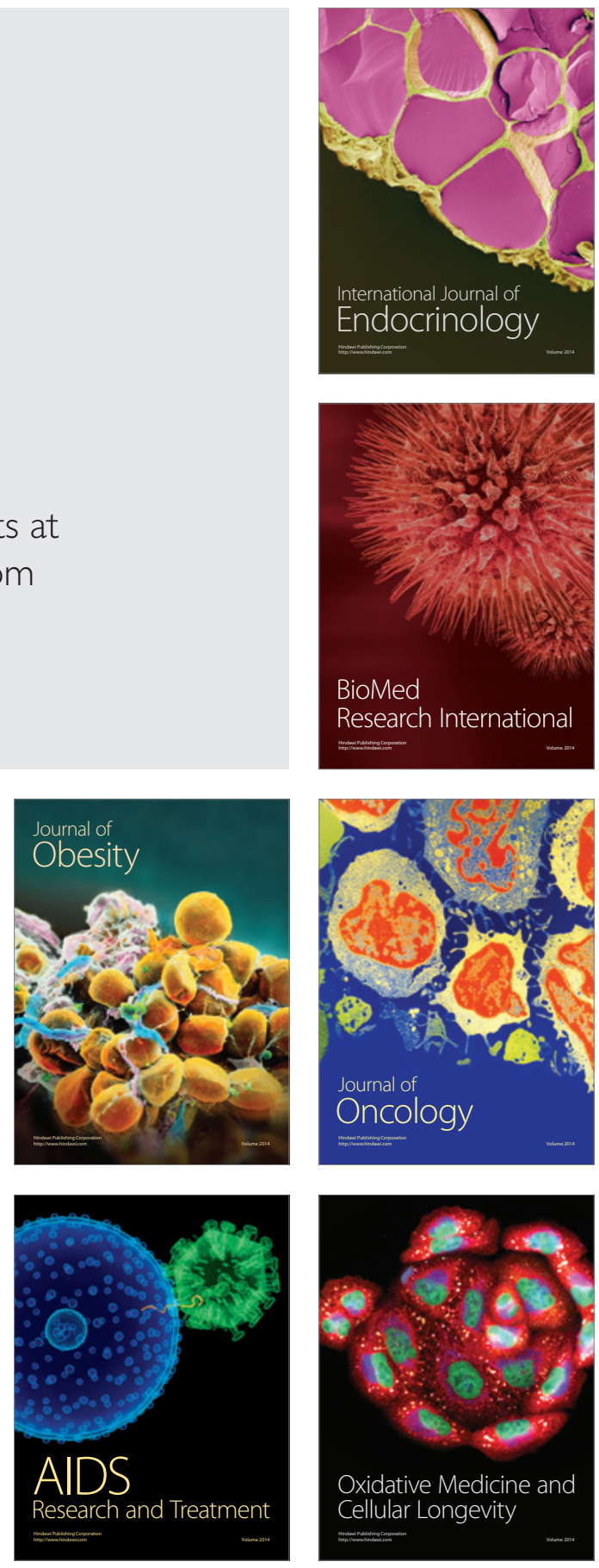